\title{
Network Delay-Weighted Least Squares Localization Algorithm with Taylor Expansion based on Acoustic Emission
}

\author{
Ying Wang, Yue Ma*, Fuzhong Bai, and Pengcheng Liu \\ College of Mechanical Engineering, Inner Mongolia University of Technology, Hohhot, 010051, China
}

\begin{abstract}
As a dynamic non-destructive testing technology, acoustic emission (AE) detection technology can locate the early damage of equipment and provide data support for health management. In order to improve the accuracy of wireless AE-based location, this paper proposes a Taylor expansion least squares algorithm to concern network delay. Firstly, according to the planar four-point positioning structure, a wireless AE detection system based on star topology is constructed, and the Taylor expanded least squares localization algorithm is then proposed. Secondly, the network queuing delay is analyzed, and the algorithm is modified with weighting factors of delay. Finally, simulation results show that the accuracy of the network delay-weighted algorithm has been improved.
\end{abstract}

Keywords: acoustic emission; network delay; localization accuracy

(Submitted on October 15, 2018; Revised on November 13, 2018; Accepted on December 14, 2018)

(C) 2019 Totem Publisher, Inc. All rights reserved.

\section{Introduction}

Acoustic emission detection technology, which was developed rapidly after the 1950s, has become a widely used nondestructive testing technology. Acoustic emission is a kind of physical phenomena of releasing strain energy in the form of elastic waves, where the material is deformed or fractured by external or internal forces. It can also be caused by inside existing or potential defects under the influence of external changes [1]. Source location, as one of the core issues in the research of acoustic emission technology, has great importance for dynamic monitoring of the materials' early damage [2].

Many researchers have studied source location technology based on acoustic emission, and several efficient and accurate algorithms have been proposed. Based on the principle of localization algorithms, the existing algorithms can be divided into two categories: distance-independent and distance-dependent [3-4]. The distance independent algorithms include the centroid positioning algorithm [5-7], Distance Vector Hops (DV-HOP) algorithm [8-10], and Approximate Point-In-Triangulation (APIT) algorithm [11-13]. The distance-dependent algorithms include the time delay estimation algorithm [14-16], least squares method [17-19], and maximum likelihood estimation [4, 20-22]. The distance-independent algorithms do not require the information of inter-node distance or included angle, but they have low accuracy and are not suitable for precise positioning. As a widely used distance-dependent estimation method, the maximum likelihood estimation is a method of estimating the statistical model parameters to maximize the likelihood function. However, the subtraction process in the distance-dependent algorithm will cause the loss of useful coordinate information, which affects the accuracy of positioning. The least squares method can avoid the loss of known coordinate information with high positioning accuracy, low power consumption, and extensive location coverage.

In practice, there are many difficulties in cabling AE equipment, especially when facing the steep terrain outdoors or complex environment of the industrial fields. For the source location of rotational machines, such as the breakage detection of wind turbine blades and the performance evaluation of aero-engines, the data of traditional AE detection is hard to transmit. In this paper, the wireless AE detection technology is concerned. However, the application of wireless network leads to new problems. The quantitative parameters of network quality of service, such as transmission delay, packet loss,

\footnotetext{
* Corresponding author.

E-mail address: my_imut_iame_0028@163.com
} 
jitter, and bit error rate, can degrade the network performance to influence the positioning accuracy. For QoS factors, transmission delay, which consists of send processing delay, queuing delay, propagation delay, and receive processing delay, is the primary factor for positioning. To the best of our knowledge, there are still no related works concerning the delay factor of wireless $\mathrm{AE}$ positioning. In this paper, the send processing delay and receive processing delay are considered to be constant, and they are related to the hardware performance of the device [23]. Since the signal speed is considered to be equal to the light speed in the air, i.e., $2 \times 10^{8} \mathrm{~m} / \mathrm{s}$, the propagation delay can thus be ignored in short-distance transmission [24].

Since all wireless nodes work in the shared medium, the network packets can only be transmitted one by one. In all the elements of network-induced delay [25], the queuing delay is the main factor affecting the accuracy of the wireless AE localization [10, 26-28]. A price-based interactive data queue management approach (PI-DQM) for delay-tolerant mobile sensor networks (DT-MSNs) is presented in paper [29] to address the priority deviation problem during the data transmission process. Paper [30] develops a continuous time Markov model to evaluate the packet sojourn time and design an expectation-maximization (EM) algorithm to calibrate the transition rate in the model. Paper [31] proposes a Channelbased Sampling rate and Queuing state Control (CSQC) scheme to minimize the packet transmission delay in industrial wireless sensor networks, and this scheme has low delay compared with the delay of IEEE 802.15.4 standard under varying interference effects. The state of the art and development directions of the queuing algorithm of WNCs are presented in paper [32]. Paper [33] proposes a method for controlling the number of OEO conversions; it uses a token bucket technique to realize the desired queuing delay performance. With this technique, the number of OEO conversions can be dynamically and freely controlled regardless of the traffic condition. Paper [34] investigates the convergence behavior and the queue delay performance of the conventional MWQ iterations in which the channel state information (CSI) and queue state information (QSI) are changing in a similar timescale as the algorithm iterations. In order to improve the accuracy of wireless AE-based location, this paper will take propagation delay caused by the priority queuing technology into consideration [35].

The rest of the paper is organized as follows: the second part of this paper deduces the derivation process of the least squares method based on Taylor expansion. In the third part, a weighting function concerning the network queuing delay is considered to improve the positioning accuracy. Finally, experimental simulations and analysis results are illustrated.

\section{Taylor Expansion of the Least Squares Algorithm}

\subsection{Least Square Positioning Principle}

Taking a lead-break signal to simulate the acoustic emission source, a planar four-point positioning method is considered in this paper, and the coordinate position of $\mathrm{AE}$ sensors are shown in Figure 1. Assume that the coordinate of the source $\mathrm{E}$ is $(x$, $y$ ) and the coordinates of the sensors $\mathrm{A}, \mathrm{B}, \mathrm{C}$, and $\mathrm{D}$ are $\left(x_{a}, y_{a}\right),\left(x_{b}, y_{b}\right),\left(x_{c}, y_{c}\right)$, and $\left(x_{d}, y_{d}\right)$, respectively. The distances between the source and the $\mathrm{AE}$ sensor are $d_{a}, d_{b}, d_{c}$, and $d_{d}$, respectively.

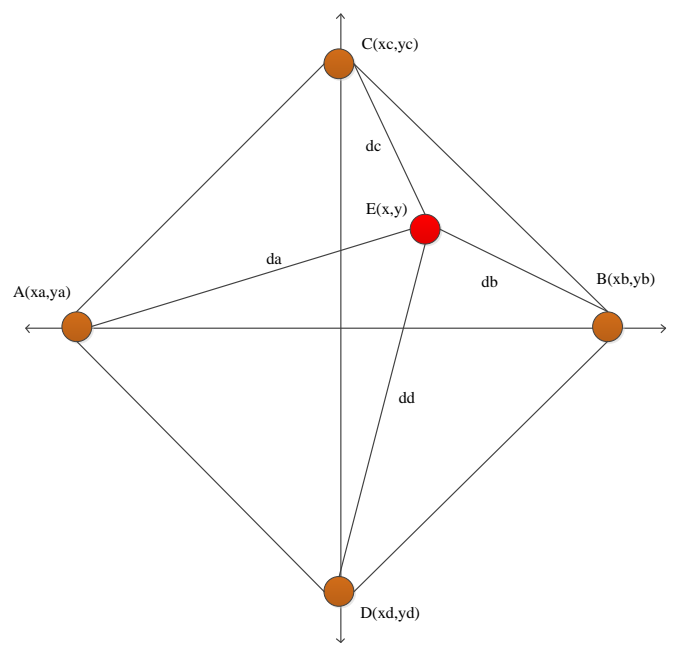

Figure 1. Four-point AE localization planar geometry graph

According to the geometry relation between the AE source and AE sensors, the following equations exist: 


$$
\left\{\begin{array}{l}
\left(x_{a}-x\right)^{2}+\left(y_{a}-y\right)^{2}=d_{a}^{2} \\
\left(x_{b}-x\right)^{2}+\left(y_{b}-y\right)^{2}=d_{b}^{2} \\
\left(x_{c}-x\right)^{2}+\left(y_{c}-y\right)^{2}=d_{c}{ }^{2} \\
\left(x_{d}-x\right)^{2}+\left(y_{d}-y\right)^{2}=d_{d}^{2}
\end{array}\right.
$$

In order to eliminate the quadratic term, the least squares method is used to solve Equation (1). Taking the first three equations successively to subtract the last one, a linear equation can be obtained as in Equation (2):

$$
A X=B
$$

Where

$$
A=2 \times\left[\begin{array}{cc}
x_{a}-x_{d} & y_{a}-y_{d} \\
x_{b}-x_{d} & y_{b}-y_{d} \\
x_{c}-x_{d} & y_{c}-y_{d}
\end{array}\right], \quad X=\left[\begin{array}{l}
x \\
y
\end{array}\right], \quad B=\left[\begin{array}{l}
x_{a}{ }^{2}-x_{d}{ }^{2}+y_{a}{ }^{2}-y_{d}{ }^{2}+d_{d}{ }^{2}-d_{a}{ }^{2} \\
x_{b}{ }^{2}-x_{d}{ }^{2}+y_{b}{ }^{2}-y_{d}{ }^{2}+d_{d}{ }^{2}-d_{b}{ }^{2} \\
x_{c}{ }^{2}-x_{d}{ }^{2}+y_{c}{ }^{2}-y_{d}{ }^{2}+d_{d}{ }^{2}-d_{c}{ }^{2}
\end{array}\right]
$$

The solution of Equation (2) is as follows:

$$
\hat{x}=\left(A^{T} A\right)^{-1} A^{T} B
$$

According to the arrival time of the AE signals, the distance $d_{i}(i=A, B, C, D)$ from the source to each sensor is calculated. By solving Equation (3), the location of the source $(x, y)$ can be roughly calculated. Because of the subtraction process, some useful coordinate information of the AE sensors will be lost to yield large errors. In the meantime, the estimation of distance $d_{i}$ is greatly affected by the accuracy of arrival time, which depends on the network induced delay. Therefore, a delay-weighted Taylor expansion algorithm is proposed in the following part to improve the localization accuracy.

\subsection{Taylor Expansion Least Squares}

In order to avoid the loss of accuracy by the subtraction process, the quadratic equation is linearized using the Taylor expansion formula.

Define the distance $d_{i}$ as follows:

$$
d_{i}=f(x, y)=\sqrt{\left(x-x_{i}\right)^{2}+\left(y-y_{i}\right)^{2}}, i=a, b, c, d
$$

$f(x, y)$ is a Taylor expansion at the point $\left(x_{0}, y_{0}\right)$ :

$$
\begin{aligned}
f(x, y) & =f\left(x_{0}+h, y_{0}+k\right) \\
& =\sqrt{\left(x_{0}-x_{i}\right)^{2}+\left(y_{0}+y_{i}\right)^{2}} \\
& +\frac{\left(x_{0}-x_{i}\right)}{\sqrt{\left(x_{0}-x_{i}\right)^{2}+\left(y_{0}+y_{i}\right)^{2}}} h \\
& +\frac{\left(y_{0}-y_{i}\right)}{\sqrt{\left(x_{0}-x_{i}\right)^{2}+\left(y_{0}+y_{i}\right)^{2}}} k+R_{n} \\
& i=a, b, c, d
\end{aligned}
$$

By eliminating the remaining items of Equation (5), Equation (1) can be deduced as 


$$
\left\{\begin{array}{l}
\frac{\left(x-x_{a}\right)}{\sqrt{\left(x-x_{a}\right)^{2}+\left(y-y_{a}\right)^{2}}} h+\frac{\left(y-y_{a}\right)}{\sqrt{\left(x-x_{a}\right)^{2}+\left(y-y_{a}\right)^{2}}} k=d_{a}-\sqrt{\left(x-x_{a}\right)^{2}+\left(y-y_{a}\right)^{2}} \\
\frac{\left(x-x_{b}\right)}{\sqrt{\left(x-x_{b}\right)^{2}+\left(y-y_{b}\right)^{2}}} h+\frac{\left(y-y_{b}\right)}{\sqrt{\left(x-x_{b}\right)^{2}+\left(y-y_{b}\right)^{2}}} k=d_{b}-\sqrt{\left(x-x_{b}\right)^{2}+\left(y-y_{b}\right)^{2}} \\
\frac{\left(x-x_{c}\right)}{\sqrt{\left(x-x_{c}\right)^{2}+\left(y-y_{c}\right)^{2}}} h+\frac{\left(y-y_{c}\right)}{\sqrt{\left(x-x_{c}\right)^{2}+\left(y-y_{c}\right)^{2}}} k=d_{c}-\sqrt{\left(x-x_{c}\right)^{2}+\left(y-y_{c}\right)^{2}} \\
\frac{\left(x-x_{d}\right)}{\sqrt{\left(x-x_{d}\right)^{2}+\left(y-y_{d}\right)^{2}}} h+\frac{\left(y-y_{d}\right)}{\sqrt{\left(x-x_{d}\right)^{2}+\left(y-y_{d}\right)^{2}}} k=d_{d}-\sqrt{\left(x-x_{d}\right)^{2}+\left(y-y_{d}\right)^{2}}
\end{array}\right.
$$

The initial value of $\left(x_{0}, y_{0}\right)$ is calculated by Equation (3). The fault coordinate will be observed using the least squares method with the aim of satisfying the following Equation:

$$
\sqrt{h^{2}+k^{2}}<\varepsilon_{\text {threshold }}
$$

Where $\varepsilon_{\text {threshold }}$ is the threshold, which depends on the maximum allowable localization error of $x$ and $y$. If Equation (7) holds, the value of $\left(x_{0}, y_{0}\right)$ is set as the coordinate of the source; otherwise, the next cycle is iterated. Let $x_{1}=x_{0}+h, y_{1}=y_{0}+k$, and repeat this process until Equation (7) is satisfied. After the $m^{\text {th }}$ iteration, the coordinate $\left(x_{m}, y_{m}\right)$ is set as the source coordinate.

\section{Taylor's Weighted Least Squares}

\subsection{Least Squares Weight Calculation Method}

To improve the accuracy of the aforementioned Taylor expansion least squares estimation, the decomposed Equation (6) is weighted with the purpose of measuring the credibility of the data detected by AE sensors.

The original $A X=B$ is replaced by $W A X=W B$, where $W$ is a weighted diagonal matrix as follows:

$$
W=\left[\begin{array}{cccc}
w_{A} & 0 & 0 & 0 \\
0 & w_{B} & 0 & 0 \\
0 & 0 & w_{C} & 0 \\
0 & 0 & 0 & w_{D}
\end{array}\right]
$$

Equation (6) can be further rewritten as

$$
\left\{\begin{array}{l}
w_{A}\left(\frac{\left(x-x_{a}\right)}{\sqrt{\left(x-x_{a}\right)^{2}+\left(y-y_{a}\right)^{2}}} h+\frac{\left(y-y_{a}\right)}{\sqrt{\left(x-x_{a}\right)^{2}+\left(y-y_{a}\right)^{2}}} k\right)=w_{A}\left(d_{a}-\sqrt{\left(x-x_{a}\right)^{2}+\left(y-y_{a}\right)^{2}}\right) \\
w_{B}\left(\frac{\left(x-x_{b}\right)}{\sqrt{\left(x-x_{b}\right)^{2}+\left(y-y_{b}\right)^{2}}} h+\frac{\left(y-y_{b}\right)}{\sqrt{\left(x-x_{b}\right)^{2}+\left(y-y_{b}\right)^{2}}} k\right)=w_{B}\left(d_{b}-\sqrt{\left(x-x_{b}\right)^{2}+\left(y-y_{b}\right)^{2}}\right) \\
w_{C}\left(\frac{\left(x-x_{c}\right)}{\sqrt{\left(x-x_{c}\right)^{2}+\left(y-y_{c}\right)^{2}}} h+\frac{\left(y-y_{c}\right)}{\sqrt{\left(x-x_{c}\right)^{2}+\left(y-y_{c}\right)^{2}}} k\right)=w_{C}\left(d_{c}-\sqrt{\left(x-x_{c}\right)^{2}+\left(y-y_{c}\right)^{2}}\right) \\
w_{D}\left(\frac{\left(x-x_{d}\right)}{\sqrt{\left(x-x_{d}\right)^{2}+\left(y-y_{d}\right)^{2}}} h+\frac{\left(y-y_{d}\right)}{\sqrt{\left(x-x_{d}\right)^{2}+\left(y-y_{d}\right)^{2}}} k\right)=w_{D}\left(d_{d}-\sqrt{\left(x-x_{d}\right)^{2}+\left(y-y_{d}\right)^{2}}\right)
\end{array}\right.
$$

The solution of the weighted least squares method can be expressed as

$$
\hat{x}=\left(A^{T} W^{T} W A\right)^{-1} A^{T} W^{T} B
$$




\subsection{Weighting Function Design}

Considering the sensitivity of the positioning algorithm to network-induced delays, a weighting function is established to consider network-induced delays. Network-induced delays refer to the total duration from the detection time of AE sensors to the receiving time of the host. They include transmission delays, queuing delays, propagation delays, and processing delays. As mentioned in the first part, it is known that queuing delays, which are considered in the following, make up the majority of network induced delays. The wireless acoustic emission positioning system in the form of star topology is shown in Figure 2:

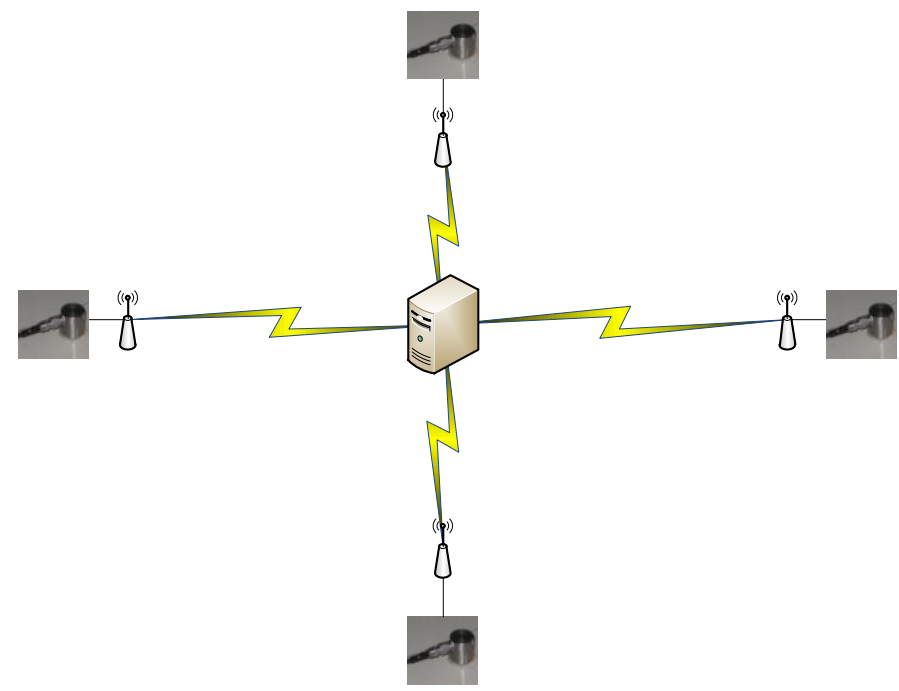

Figure 2. AE network topology graph

Since the communication medium is shared, the AE sensors A, B, C, and D transmit the detected data to the host one by one. This requires that the transmission can only be executed under a priority. Non-preemptive priority, that is, when lowpriority data is forwarded and high-priority data arrives, must wait until low-priority data is sent before forwarding the highpriority data [9]. Assume that the data in sensor $i(i=A, B, C$, and $D)$ is divided into $s$ data packets and sent to the acoustic emission host in unit time. Suppose that the transmission time of each packet is $T_{i k}(k=1,2,3, \cdots, s)$ and the transmission rate is $v$. The sending rate of each sensor is the same, and thus $v$ is constant. The average value of each packet's transmission time is calculated by Equation (11).

$$
\overline{T_{i}}=E\left\{T_{i k}\right\}=\frac{1}{v}
$$

Suppose that each acoustic emission sensor has $s$ data packets with priorities from low to high for sensors A, B, C, and $\mathrm{D}$, in unit time, respectively. The average transmission time $T_{i k}$ for each sensor is shown in Figure 3.

In Figure $3, \mathrm{D}_{i}(i=1,2,3,4,5)$ represents the transmission delay of the $i^{\text {th }}$ packet of sensor $\mathrm{D}, \mathrm{C}_{i}(i=1,2,3,4,5)$ represents the transmission delay of the $i^{\text {th }}$ packet of sensor $\mathrm{C}, \mathrm{B}_{i}(i=1,2,3,4,5)$ represents the transmission delay of the $i^{\text {th }}$ packet of sensor $\mathrm{B}$, and $\mathrm{A}_{i}(i=1,2,3,4,5)$ represents the transmission delay of the $i^{\text {th }}$ packet of sensor $\mathrm{A}$.

As shown in Section 1, we consider the queuing delay as the transmission delay for this wireless network with star topology. Since the transmission medium air is shared by all the nodes of this network, the packet of each AE sensor has to wait for transmission until that the channel is free with the guidance of the priority scheme. As in Equation (11), the transmission time of sensor $\mathrm{D}$ means that the queuing time until the last packet is able to be transmitted. For sensor $\mathrm{C}$ with lower priority, its transmission time includes two parts, the transmission of $s$ packets of sensor D and the transmission time of the first four packets of sensor C. This is formulated in Equation (12). The transmission times of sensors A and B follow the same principle as sensor C, which is shown in Equations (13) and (14).

The average waiting time of sensor $\mathrm{D}$ is 


$$
T_{D s}=\eta_{1} \overline{T_{D}}+\eta_{2} \overline{T_{D}}+\cdots+\eta_{(s-1)} \overline{T_{D}}
$$

The average wait time of sensor $\mathrm{C}$ is

$$
T_{C s}=T_{D s}+\eta_{s} \overline{T_{D}}+\eta_{1} \overline{T_{C}}+\eta_{2} \overline{T_{C}}+\cdots+\eta_{(s-1)} \overline{T_{C}}
$$

The average waiting time of sensor B is

$$
T_{B s}=T_{C s}+\eta_{s} \overline{T_{C}}+\eta_{1} \overline{T_{B}}+\eta_{2} \overline{T_{B}}+\cdots+\eta_{(s-1)} \overline{T_{B}}
$$

The average waiting time of sensor $\mathrm{A}$ is

$$
T_{A s}=T_{B s}+\eta_{s} \overline{T_{B}}+\eta_{1} \overline{T_{A}}+\eta_{2} \overline{T_{A}}+\cdots+\eta_{(s-1)} \overline{T_{A}}
$$

Where $\eta_{k}=\frac{\alpha_{i k}}{v}$ is the waiting intensity and $\alpha_{i k}$ is the number of packets to be sent in unit time for the $i^{\text {th }}$ sensor.

The total occupation time of the medium for the $i^{\text {th }}$ sensor is the average waiting of this sensor $T_{s i}$ plus the transmission time of one packet $\overline{T_{i k}}$, that is,

$$
H_{i}=T_{s i}+\overline{T_{i s}}
$$

The delay induced weighting factor is defined as follows:

$$
W_{i}=\frac{1}{H_{i}}
$$

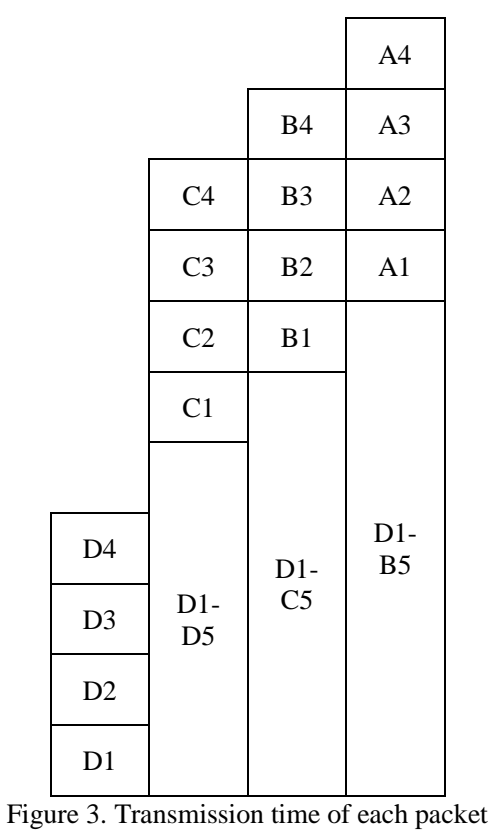

\section{Experiment Analysis}

In order to verify the effectiveness of the proposed weighted least squares localization algorithm in this paper, a simulation 
experiment is conducted. By taking a lead-break signal to simulate an AE source signal, all the data are detected by $R 3 \alpha$ AE sensors, preamp, and PCI-2 acquisition card (such as Figure 4) of Physical Acoustic Corporation (PAC). The resonant frequency is $90 \mathrm{kHz}$, and the operating frequency range is $1 \sim 100 \mathrm{kHz}$. The AE threshold is set to $40 \mathrm{~dB}$, and the sampling frequency is $4 \mathrm{MHz}$. The lead breaking experiment is executed on a square iron plate with a width of 500mm. Four AE sensors are arranged as shown in Figure 5 in the experiment, and the coordinates are defined as A $(-250,0), \mathrm{B}(250,0), \mathrm{C}(0$, $250)$, and $\mathrm{D}(0,-250)$ with the unit of $\mathrm{mm}$.

PAC's acoustic emission sensors are available in a variety of models from sensor size, center frequency, and interface form. The $R 3 \alpha$ sensor has a size of $19 \mathrm{~mm} \times 22 \mathrm{~mm}$, and the center frequency is $30 \mathrm{kHz}$.

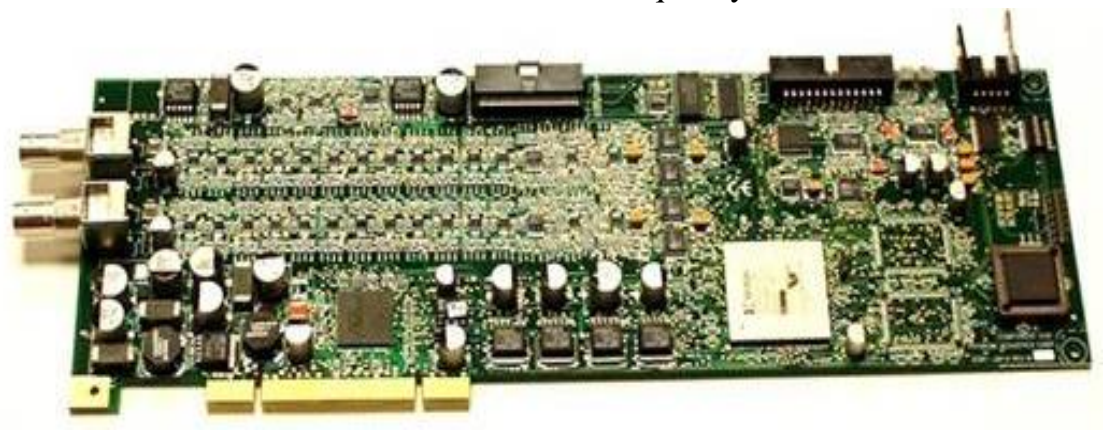

Figure 4. PCI-2 acquisition card

PCI-2 has an 18-bit A/D, and the frequency range is $1 \sim 3 \mathrm{kHz}$. It is able to store detection data into the hard disk continuously with a speed of $10 \mathrm{Mbps}$.

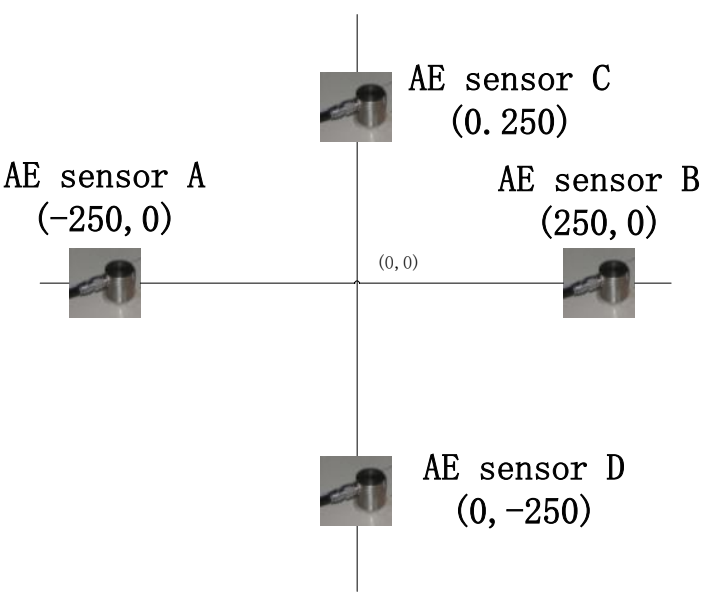

Figure 5. AE sensors arrangement graph

AE sensors A, B, C, and D have priorities from low to high. With the data sampling time of $0.25 \mathrm{~ms}$ for each sensor, the data size is up to $336 \mathrm{M}$ bps. According to the network protocol, the data is divided into five packets to transmit. The transmission rate $v$ is 6 units/ $\mu \mathrm{s}$, and the transmission time $\overline{T_{i k}}$ is $0.17 \mu \mathrm{s} /$ units. The queuing parameters for the four sensors are shown in Figure 6.

The detailed data in Figure 6 is shown in Table 1.

The delays of the four sensors according to Equations (10) to (15) are shown in Table 2.

According to the average queuing delay of each sensor, the weighting matrix $W$ is expressed as

$$
W=\left[\begin{array}{cccc}
0.183 & 0 & 0 & 0 \\
0 & 0.327 & 0 & 0 \\
0 & 0 & 0.738 & 0 \\
0 & 0 & 0 & 2.725
\end{array}\right]
$$




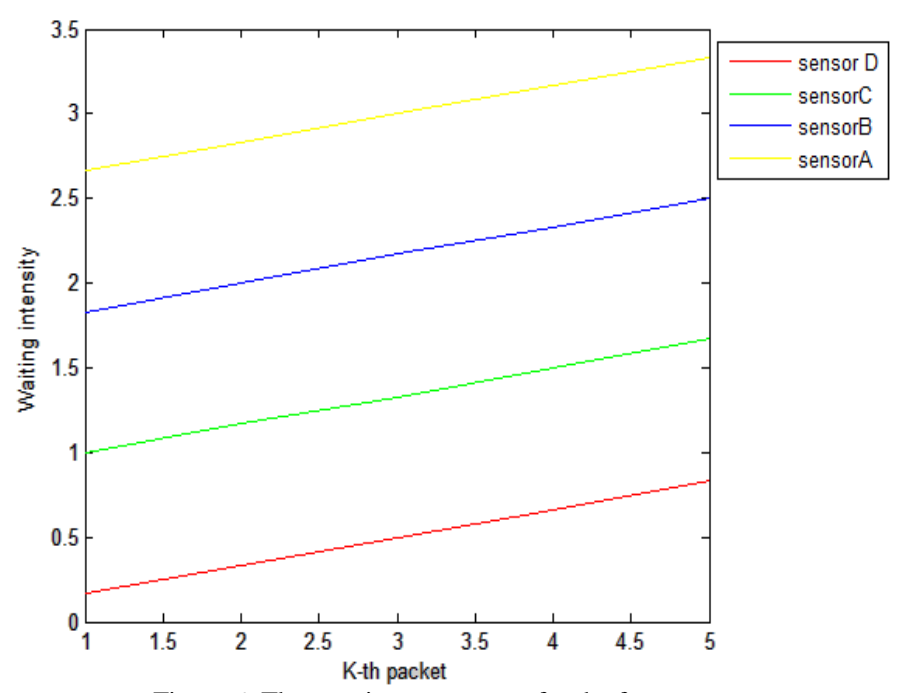

Figure 6. The queuing parameters for the four sensors

\begin{tabular}{|c|c|c|c|c|}
\multicolumn{5}{|c|}{ Table 1. Waiting intensity of the $i^{\text {th }}$ sensor and $k^{\text {th }}$ packet } \\
\hline$k^{i}$ & Sensor A & Sensor B & Sensor C & Sensor D \\
\hline 2 & 2.67 & 1.83 & 1 & 0.17 \\
\hline 3 & 2.83 & 2 & 1.17 & 0.33 \\
\hline 4 & 3.17 & 2.17 & 1.33 & 0.5 \\
\hline 5 & 3.33 & 2.5 & 1.5 & 0.66 \\
\hline
\end{tabular}

Table 2. Calculation results of each sensor's delay

\begin{tabular}{|c|c|c|}
\hline Sensor & $T_{i}$ & $H_{i}$ \\
\hline Sensor A & 5.294 & 5.464 \\
\hline Sensor B & 2.885 & 3.055 \\
\hline Sensor C & 1.185 & 1.355 \\
\hline Sensor D & 0.197 & 0.367 \\
\hline
\end{tabular}

The maximum allowable errors of $x$ and $y$ are set as $20 \mathrm{~mm}$. The proposed localization algorithm is simulated in MATLAB software. The experiments are carried out with the lead-break signals at the coordinates of $(0,0),(10,0)$, and $(60,0)$ with the unit of $\mathrm{mm}$. Compare the weighted positioning algorithm with the unweighted one using the following formula:

$$
\frac{\sqrt{\left(\Delta x^{2}+\Delta y^{2}\right)}}{500} \times 100 \%=\text { Relative error }
$$

Where $\Delta x$ and $\Delta y$ are the error of coordinates between the calculated point and the lead-break point. The results are shown in Figure 7.

The absolute error and relative error are shown in Table 3.

As seen from Table 3, the accuracy of the weighted positioning algorithm is higher than that of the unweighted positioning algorithm. There are two main sources in the error analysis:

- Placement error of the acoustic emission sensors, which may affect the positioning accuracy greatly in the case of short-distance AE detection.

- Coordinate error of the stimulated source. The coordinate of lead break as a stimulated source is carried out by hand to influence the accuracy. 


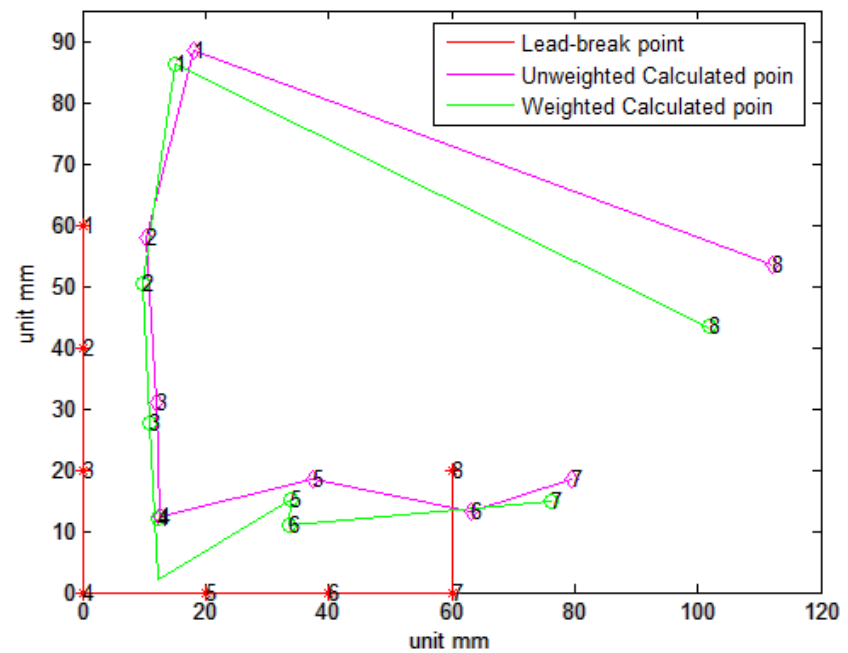

Figure 7. Comparison of weighted and unweighted positioning algorithms

Table 3. Comparison results of weighted and unweighted algorithms

\begin{tabular}{|c|c|c|c|c|}
\hline Positioning method & Lead-break point & Calculated point & Absolute error & Relative error \\
\hline \multirow{8}{*}{$\begin{array}{l}\text { Weighted positioning } \\
\text { algorithm }\end{array}$} & $(0,60)$ & $(15.1,86.2)$ & $(15.1,26.2)$ & $6.1 \%$ \\
\hline & $(0,40)$ & $(9.8,50.5)$ & $(9.8,10.5)$ & $2.9 \%$ \\
\hline & $(0,20)$ & $(10.8,27.7)$ & $(10.8,7.7)$ & $2.7 \%$ \\
\hline & $(0,0)$ & $(12.4,12.2)$ & $(12.4,12.2)$ & $3.5 \%$ \\
\hline & $(20,0)$ & $(33.9,15.1)$ & $(13.9,15.1)$ & $4.1 \%$ \\
\hline & $(40,0)$ & $(33.7,10.9)$ & $(6.3,10.9)$ & $2.6 \%$ \\
\hline & $(60,0)$ & $(76.1,14.9)$ & $(16.1,14.9)$ & $4.4 \%$ \\
\hline & $(60,20)$ & $(101.8,43.3)$ & $(41.8,23.3)$ & $9.6 \%$ \\
\hline Positioning method & Lead-break point & Calculated point & Absolute error & Relative error \\
\hline \multirow{8}{*}{$\begin{array}{l}\text { Unweighted } \\
\text { positioning algorithm }\end{array}$} & $(0,60)$ & $(18.1,88.6)$ & $(18.1,28.6)$ & $6.8 \%$ \\
\hline & $(0,40)$ & $(10.5,57.9)$ & $(10.5,17.9)$ & $4.2 \%$ \\
\hline & $(0,20)$ & $(11.9,30.9)$ & $(11.9,10.9)$ & $3.2 \%$ \\
\hline & $(0,0)$ & $(12.5,12.5)$ & $(12.5,12.5)$ & $3.5 \%$ \\
\hline & $(20,0)$ & $(37.4,18.6)$ & $(17.4,18.6)$ & $5.1 \%$ \\
\hline & $(40,0)$ & $(63.2,13.1)$ & $(23.2,13.1)$ & $5.3 \%$ \\
\hline & $(60,0)$ & $(79.6,18.5)$ & $(19.6,18.5)$ & $5.4 \%$ \\
\hline & $(60,20)$ & $(112,53.5)$ & $(52,13.5)$ & $10.7 \%$ \\
\hline
\end{tabular}

\section{Conclusions}

Acoustic emission is one of the most widely used non-destructive testing techniques, and its high positioning accuracy has received extensive attention in the field. Based on the least squares method, the reliability of each $\mathrm{AE}$ sensor and the corresponding data are weighted concerning the network induced delay, and experiments are executed to verify the proposed algorithm. The results show that the modified algorithm with weighting factors can effectively improve the accuracy of AE localization.

In the future, other factors of network delay with different network protocols and topologies will be considered, and the deep research of wireless transmission delay mechanisms will also be investigated.

\section{Acknowledgements}

This work is supported by the School Fund of IMUT: Reliability Study of Wireless Networked Control System (ZD201503) 
and the Fault Diagnosis Research of the Networked Control System of High-Speed Train (NJZZ16085).

\section{References}

1. W. Y. Hong and J. Yi, "Maximum Likelihood Network Localization using Range Estimation and GPS Measurements," in Proceedings of 2017 9th International Conference on Wireless Communications and Signal Processing (WCSP), pp. 1-6, 2017

2. M. Baz and P. D. Mitchell, "Analysis of Queuing Delay and Medium Access Distribution over Wireless Multihop PANs," IEEE Transactions on Vehicular Technology, Vol. 64, No. 7, pp. 2972-2990, 2015

3. X. L. Chen and J. Yong, "AUV Underwater Terrain Matching Location Method based on Maximum Likelihood Estimation," Robot, Vol. 34, No. 5, pp. 559-565, 2012

4. B. R. Ahmad and Z. I. A. Khalib, "High Deadline Meeting Rate of Non-Preemptive Dynamic Soft Real Time Scheduling Algorithm," in Proceedings of 2012 IEEE International Conference on Control System, Computing and Engineering, pp. 296301,2012

5. A. Giorgetti and K. Magowe, "Exact Analysis of Weighted Centroid Localization," in Proceedings of 201624 th European Signal Processing Conference (EUSIPCO), pp. 743-747, 2016

6. N. Iiyama and T. Shimada, "Dynamic Control Method of Queuing Delay with/without OEO Conversion in a Multi-Stage Access Network," in Proceedings of 2012 World Telecommunications Congress, pp. 1-6, 2012

7. L. Pantera and O. I. Traore, "Reproducible Data Processing Research for The CABRI R.I.A. Experiments Acoustic Emission Signal Analysis," in Proceedings of 2015 4th International Conference on Advancements in Nuclear Instrumentation Measurement Methods and their Applications (ANIMMA), pp. 1-8, 2015

8. Y. Liu, “An Improved APIT Location Algorithm for Wireless Sensor Networks," Journal of Liaodong University, Vol. 20 , No. 2, pp. 115-119, 2013

9. X. Lin and S. Kumar, "AFCD: An Approximated-Fair and Controlled-Delay Queuing for High Speed Networks," in Proceedings of 2013 22nd International Conference on Computer Communication and Networks (ICCCN), pp. 1-7, 2013

10. X. H. Chen and J. Peng, "Centroid Location Algorithm for Wireless Sensor Networks based on Optimal Beacon Nodes," Journal of Computer Applications, Vol. 35, No. 1, pp. 5-9, 2015

11. H. Huo and Q. Q. Shi, "Using the Steepest Descent Algorithm to Improve the Node Location Accuracy of the Maximum Likelihood Estimation Algorithm," Application Research of Computers, Vol. 25, No. 7, pp. 38-40, 2008

12. S. Y. Jing, "Generalized Cross Correlation Time Delay Estimation Sound Localization Algorithm," Northwestern Polytechnical University, Vol. 33, No. 5, pp. 464-468, 2014

13. Y. H. Li and L. M. Yang, "Analysis of Time Delay in Networked Control Systems and Study of Data Transmission Technology," Control and Decision, pp. 361-382, 2008

14. C. J. Sun and L. J. Li, "Quadratic Correlation Time Delay Estimation Algorithm based on Kaiser Window and Hilbert Transform," in Proceedings of 2016 Sixth International Conference on Instrumentation \& Measurement, Computer, Communication and Control (IMCCC), pp. 927-931, 2016

15. Q. S. Yu and F. W. Zeng, "Two-Stage Centroid Localization for Wireless Sensor Networks using Received Signal Strength," in Proceedings of 2016 19th International Conference on Network-based Information Systems, pp. 370-375, 2016

16. K. Zhang and Q. H. Li, "Channel-based Sampling Rate and Queuing State Control in Delay-Constraint Industrial WSNs," in Proceedings of GLOBECOM 2017 - 2017 IEEE Global Communications Conference, pp. 1-6, 2017

17. Z. H. Gu and X. P. Wu, "A Linear Least Square Method for Sound Source Target Precise Localization," ACTA ACAI SINICA, Vol. 41, No. 1, pp. 87-93, 2016

18. H. Tall and G. Chalhoub, "ComLoB: Link Quality and Queuing Delay based Composite Routing Protocol for Traffic Load Balancing in WSNs," in Proceedings of 2018 IEEE Middle East and North Africa Communications Conference, pp. 1-6, 2018

19. Y. Zhang and Z. L. Zhu, "A Novel DV-Hop Method for Localization of Network Nodes," in Proceedings of 201636 th Chinese Control Conference (CCC), pp. 8346-8351, 2016

20. H. Huang and W. S. Fang, "DV-HOP Triple Localization Algorithm," Journal of Jiang Xi University of Science and Technology, Vol. 38, No. 3, pp. 92-96, 2017

21. L. Peng and W. Zhang, "Based on The Cyclic Refinement APIT Localization Algorithm for Wireless Sensor Networks," in Proceedings of the 29th Chinese Control Conference, pp. 4753-4756, 2010

22. X. H. Huang and Y. B. Zhang, "Study of Positioning of Rock Acoustic Emission Source based on Phase Difference Time Delay Estimation," Rock and Soil Mechanics, Vol. 36, No. 2, pp. 382-386, 2015

23. Q. Z. Mao and H. Z. Zhuang, "DV-HOP Localization Algorithm with Weight-based Oriented Cuckoo Search Algorithm," in Proceedings of 2017 36th Chinese Control Conference (CCC), pp. 2534-2539, 2017

24. Z. H. Xu and P. T. Yu, "Localization in Sensor Networks with Communication Delays and Package Losses," in Proceedings of 2017 IEEE 56th Annual Conference on Decision and Control (CDC), pp. 3974-3979, 2017

25. J. Li and Q. Y. Li, "A Price-based Interactive Data Queue Management Approach for Delay-Tolerant Mobile Sensor Networks," in Proceedings of 2013 IEEE Wireless Communications and Networking Conference Workshops (WCNCW), pp. 189-194, 2013

26. J. Salzmann and R. Behnke, "SDLSne - Improved Scalable Distributed Least Squares Localization with Minimized Communication," in Proceedings of 21st Annual IEEE International Symposium on Personal, Indoor and Mobile Radio Communications, pp. 1695-1700, 2010

27. X. B. Huang and Y. H. Hu, "Online Particle Size Measurement Through Acoustic Emission Detection and Signal Analysis," in Proceedings of 2014 IEEE International Instrumentation and Measurement Technology Conference (I2MTC), pp. 949-953, 2014

28. A. Singh and S. Jain, "Improved APIT Localization Algorithm in Wireless Sensor Networks," in Proceedings of 2017 4th 
International Conference on Signal Processing, Computing and Control (ISPCC), pp. 77-81, 2017

29. J. Wang and F. Yan, "A Bluetooth Location Method based on Least Squares," Computer Networks and Networks, Vol. 19, pp. 72-74, 2017

30. J. T. Chen and V. K. N. Lau, "Delay Analysis of Max-Weight Queue Algorithm for Time-Varying Wireless Ad hoc Networks - Control Theoretical Approach," IEEE Transactions on Signal Processing, Vol. 61, No. 1, pp. 99-108, 2013

31. J. M. Pietraszko and J. Tancula, "Computer Networks Stability Independence of the Queuing Delays," in Proceedings of Fifth International Conference on the Innovative Computing Technology (INTECH 2015), pp. 118-123, 2015

32. A. H. A. Halim and R. B. Ahmad, "Performance Analysis of Throughput and Queuing Delay for Multiple Optical Channels for Local Area Network," in Proceedings of 2014 5th International Conference on Intelligent Systems, Modelling and Simulation, pp. 221-224, 2014

33. C. W. Qu and Z. Xu, "Novel Passive Localization Algorithm based on Double Side Matrix-Restricted Total Least Squares," Chinese Journal of Aeronautics, Vol. 26, No. 4, pp. 1008-1016, 2013

34. F. W. Xue and Q. W. Yuan, "Locating The Position of Objects in Non-Line-of-Sight based on Time Delay Estimation," Chinese Physics B, No. 8, pp. 30-37, 2016 